Supporting Information

\title{
Extended Gibbs free energy and Laplace pressure of ordered Hexagonal Close Pack spherical particles: Wettability Study
}

Amir Bayat $^{\mathrm{a}}$, Mahdi Ebrahimi ${ }^{\mathrm{b}}$, Saeed Rahemi Ardekanic ${ }^{\mathrm{c}}$ Esmaiel Saievar Iranizada,*, Alireza Zaker Moshfegh ${ }^{\mathrm{b}, *}$

a Department of Basic Science, Tarbiat Modares University, P.O. Box 14115-111, Tehran, Iran

${ }^{b}$ Department of Physics, Sharif University of Technology, P.O. Box 11155-9161, Tehran, Iran

${ }^{c}$ Department of Engineering, Tarbiat Modares University, P.O. Box 14115-111, Tehran, Iran

\section{Appendix 1}

Equation 21 can be derived from equations 18,19, and 20 as

$$
\begin{aligned}
& m_{S}-m_{L}=\frac{-a}{\sqrt{h^{2}-a^{2}}}-\left(\frac{a-h}{\sqrt{r^{2}-(a-h)^{2}}}\right)=\left(\frac{-a \sqrt{r^{2}-(a-h)^{2}}+(h-a) \sqrt{h^{2}-a^{2}}}{\sqrt{h^{2}-a^{2}} \sqrt{r^{2}-(a-h)^{2}}}\right) \\
& 1+m_{S} m_{L}=1-\left(\frac{a(a-h)}{\sqrt{h^{2}-a^{2}} \sqrt{r^{2}-(a-h)^{2}}}\right)=\left(\frac{\sqrt{h^{2}-a^{2}} \sqrt{r^{2}-(a-h)^{2}}-a(a-h)}{\sqrt{h^{2}-a^{2}} \sqrt{r^{2}-(a-h)^{2}}}\right) \\
& \tan \left(\theta_{0}\right)=\frac{\left|m_{S}-m_{L}\right|}{1+m_{S} m_{L}}=\left(\frac{-a \sqrt{r^{2}-(a-h)^{2}}+(h-a) \sqrt{h^{2}-a^{2}}}{\sqrt{h^{2}-a^{2}} \sqrt{r^{2}-(a-h)^{2}}-a(a-h)}\right)
\end{aligned}
$$

using the relation between $\cos \theta$ and $\tan \theta$

$$
\begin{aligned}
& \cos ^{2}\left(\theta_{0}\right)=\frac{1}{1+\tan ^{2}\left(\theta_{0}\right)} \\
& 1+\tan ^{2}\left(\theta_{0}\right)=\left(\frac{\left[\sqrt{h^{2}-a^{2}} \sqrt{r^{2}-(a-h)^{2}}-a(a-h)\right]^{2}+\left[-a \sqrt{r^{2}-(a-h)^{2}}+(h-a) \sqrt{h^{2}-a^{2}}\right]^{2}}{\left[\sqrt{h^{2}-a^{2}} \sqrt{r^{2}-(a-h)^{2}}-a(a-h)\right]^{2}}\right)
\end{aligned}
$$

numerator of the above equation can be simplified as

$$
\begin{aligned}
& {\left[\sqrt{h^{2}-a^{2}} \sqrt{r^{2}-(a-h)^{2}}-a(a-h)\right]^{2}+\left[-a \sqrt{r^{2}-(a-h)^{2}}+(h-a) \sqrt{h^{2}-a^{2}}\right]^{2}} \\
& =\left(h^{2}-a^{2}\right)\left(r^{2}-(a-h)^{2}\right)+a^{2}(a-h)^{2}-2 a(a-h) \sqrt{h^{2}-a^{2}} \sqrt{r^{2}-(a-h)^{2}} \\
& +a^{2}\left(r^{2}-(a-h)^{2}\right)+(h-a)^{2}\left(h^{2}-a^{2}\right)+2 a(a-h) \sqrt{h^{2}-a^{2}} \sqrt{r^{2}-(a-h)^{2}}= \\
& \left(h^{2}-a^{2}\right)\left[r^{2}-(a-h)^{2}+(h-a)^{2}\right]+a^{2}\left[(a-h)^{2}+r^{2}-(a-h)^{2}\right]= \\
& h^{2} r^{2}-a^{2} r^{2}+a^{2} r^{2}=h^{2} r^{2}
\end{aligned}
$$

Thus, the equation 21 is obtained as 


$$
\begin{aligned}
& \cos ^{2}\left(\theta_{0}\right)=\frac{1}{1+\tan ^{2}\left(\theta_{0}\right)}=\frac{\left[\sqrt{h^{2}-a^{2}} \sqrt{r^{2}-(a-h)^{2}}-a(a-h)\right]^{2}}{h^{2} r^{2}} \\
& h r \cos \left(\theta_{0}\right)=\sqrt{h^{2}-a^{2}} \sqrt{r^{2}-(a-h)^{2}}-a(a-h)
\end{aligned}
$$

\section{Appendix 2}

The equation 23 can be derived from equations 21 and 22, as

$$
\begin{aligned}
& \frac{2 h \gamma \cos \left(\theta_{0}\right)}{P}=\sqrt{h^{2}-a^{2}} \sqrt{\left(\frac{2 \gamma}{P}\right)^{2}-(a-h)^{2}}-a(a-h) \\
& 2 h \gamma \cos \left(\theta_{0}\right)=\sqrt{h^{2}-a^{2}} \sqrt{(2 \gamma)^{2}-P^{2}(a-h)^{2}}-P a(a-h) \\
& {\left[2 h \gamma \cos \left(\theta_{0}\right)+P a(a-h)\right]^{2}=\left(h^{2}-a^{2}\right)\left(4 \gamma^{2}-P^{2}(a-h)^{2}\right)} \\
& 4 h^{2} \gamma^{2} \cos ^{2}\left(\theta_{0}\right)+P^{2} a^{2}(a-h)^{2}+4 h \gamma \cos \left(\theta_{0}\right) P a(a-h)-4 \gamma^{2}\left(h^{2}-a^{2}\right)+(a-h)^{2}\left(h^{2}-a^{2}\right) P^{2}=0 \\
& {\left[a^{2}(a-h)^{2}+(a-h)^{2}\left(h^{2}-a^{2}\right)\right] P^{2}+\left[4 h \gamma \cos \left(\theta_{0}\right) a(a-h)\right] P+4\left[h^{2} \gamma^{2} \cos ^{2}\left(\theta_{0}\right)-\gamma^{2}\left(h^{2}-a^{2}\right)\right]=0} \\
& {\left[h^{2}(a-h)^{2}\right] P^{2}+\left[4 h \gamma \cos \left(\theta_{0}\right) a(a-h)\right] P+4\left[h^{2} \gamma^{2} \cos ^{2}\left(\theta_{0}\right)-\gamma^{2}\left(h^{2}-a^{2}\right)\right]=0}
\end{aligned}
$$

this is a quadratic equation for $P$, thus we can write

$$
\begin{aligned}
& a x^{2}+b x+c=0 \\
& x_{1,2}=\frac{-b \pm \Delta}{2 a}=\frac{-b \pm \sqrt{b^{2}-4 a c}}{2 a} \\
& \Delta=\sqrt{b^{2}-4 a c}=\sqrt{16\left[h^{2} \gamma^{2} \cos ^{2}\left(\theta_{0}\right) a^{2}(a-h)^{2}-\gamma^{2}(a-h)^{2} h^{2}\left(h^{2} \cos ^{2}\left(\theta_{0}\right)-\left(h^{2}-a^{2}\right)\right)\right]} \\
& =\sqrt{16 h^{2} \gamma^{2}(a-h)^{2}\left[\cos ^{2}\left(\theta_{0}\right) a^{2}-h^{2} \cos ^{2}\left(\theta_{0}\right)+\left(h^{2}-a^{2}\right)\right]} \\
& =\sqrt{16 h^{2} \gamma^{2}(a-h)^{2}\left(h^{2}-a^{2}\right)\left[1-\cos ^{2}\left(\theta_{0}\right)\right]}=4 h \gamma(a-h) \sin \left(\theta_{0}\right) \sqrt{\left(h^{2}-a^{2}\right)}
\end{aligned}
$$

and finally the equation of pressure is obtained as

$$
\begin{aligned}
& P=\frac{-b \pm \Delta}{2 a}=\frac{-h \gamma \cos \left(\theta_{0}\right) a(a-h) \pm 2 h \gamma(a-h) \sin \left(\theta_{0}\right) \sqrt{\left(h^{2}-a^{2}\right)}}{(a-h)^{2} h^{2}} \\
& P=\frac{\gamma}{h(a-h)}\left(-a \cos \left(\theta_{0}\right) \pm 2 \sqrt{h^{2}-a^{2}} \sin \left(\theta_{0}\right)\right)
\end{aligned}
$$

